\title{
Molecular Dynamics Study of the Particle Size Dependency of Structural Change in Hydrogenated Model f.c.c. Nanoparticles
}

\author{
Hiroshi Ogawa \\ National Institute of Advanced Industrial Science and Technology (NRI, AIST), Tsukuba 305-8568, Japan
}

Hydrogen absorption in f.c.c. nanoparticles of 1-8 nm diameter was investigated using molecular dynamics simulation with model interatomic potentials. Atomic configuration with five-fold symmetries was observed in both hydrogen-free and hydrogenated particles smaller than $2 \mathrm{~nm}$. The f.c.c. structure was maintained in larger particles after hydrogenation in cases where the M-H interaction is weak. Lattice deformation was induced in cases of strong $\mathrm{M}-\mathrm{H}$ interaction. A shift of critical size for icosahedral-cubic transition by hydrogenation was also inferred. The number of absorbed $\mathrm{H}$ atoms increased concomitantly with increasing particle size and $\mathrm{M}-\mathrm{H}$ interaction. Most absorbed $\mathrm{H}$ atoms located at $\mathrm{O}$-sites when $\mathrm{M}-\mathrm{H}$ interaction was weak. The T-site occupancy increased with $\mathrm{M}-\mathrm{H}$ interaction. Analysis using local atomic configuration revealed that structural variation in nanoparticles results from three factors: surface effects, icosahedral transformation, and lattice deformation attributable to $\mathrm{M}-\mathrm{H}$ interaction. [doi:10.2320/matertrans.MA201017]

(Received October 13, 2010; Accepted November 11, 2010; Published January 13, 2011)

Keywords: hydrogen storage, nanoparticle, molecular dynamics, icosahedral symmetry, lattice deformation, size dependency

\section{Introduction}

Large surface area per volume of metallic nanoparticles is a favorable property for high absorption and desorption rates in hydrogen storage. Hydrogen absorption in nanoparticles has been studied for many years, mainly on $\mathrm{Pd} .{ }^{1-11)}$ Pundt and coworkers $^{6,7)}$ showed that the crystal structure of hydrogenated Pd nanoparticles varies depending on the cluster size. It includes not only the equilibrium $\alpha$ and $\alpha^{\prime}$ phases in the f.c.c. structure, but also the icosahedral phase, which was also found in hydrogenated $\mathrm{Co}$ and $\mathrm{Ni}$ nanoparticles. ${ }^{8)}$ Behaviors of $\mathrm{H}$ atoms in lattice defects such as surface, interface, vacancy and dislocation have been pointed out as key issues in hydrogen storage. ${ }^{9)}$ Yamauchi et al. ${ }^{10,11)}$ described cluster size dependent $\mathrm{P}-\mathrm{C}$ isotherms of $\mathrm{Pd}$ nanoparticles in comparison with those in bulk samples. They reported that the large difference between nanoparticles and bulk resulted from surface effects. These results show that structural variation by hydrogenation and its particle size dependency are important to understand hydrogen storage mechanisms in nanoparticles.

Aside from experimental methods, classical molecular dynamics (MD) simulation is a powerful tool for investigating structural variation in complex systems such as mixtures of nanoparticles and hydrogen atoms. Pundt et al. ${ }^{7)}$ simulated the pair distribution functions in nanoparticles using classical $\mathrm{MD}$ and compared those results with X-ray diffraction data. The present author recently conducted classical MD simulation on hydrogen absorption in metallic nanoparticles. ${ }^{12-15)}$ Previous studies have addressed the radial distribution of absorbed hydrogen atoms in nanoparticles, ${ }^{12}$ grain boundary generation by hydrogenation, ${ }^{13,14)}$ and simulated $\mathrm{P}-\mathrm{C}$ isotherms. ${ }^{15)}$ These studies were intended to elucidate mechanisms of hydrogen storage in nanoparticles on the concept of 'parameter physics' covering a wide phenomenological range. In this study, the author analyzes particle size dependencies of lattice structures and hydrogen absorption sites in model f.c.c. nanoparticles.
Table 1 Six model nanoparticles used for this study.

\begin{tabular}{ccccc}
\hline diameter $/ \mathrm{nm}$ & no. of atoms & initial shape & initial lattice & relaxed lattice \\
\hline 1 & 55 & cuboctahedron & f.c.c. & icosa. \\
1.4 & 135 & sphere & f.c.c. & icosa. \\
2 & 381 & sphere & f.c.c. & f.c.c. \\
4 & 3055 & sphere & f.c.c. & f.c.c. \\
6 & 10185 & sphere & f.c.c. & f.c.c. \\
8 & 24499 & sphere & f.c.c. & f.c.c. \\
\hline
\end{tabular}

\section{MD Simulation}

For simulating f.c.c. nanoparticles, six model particles presented in Table 1 were assumed. The smallest model comprises 55 atoms in cuboctahedral shape, of which diameter $D$ is about $1 \mathrm{~nm}$. Actually, 55 is widely known as a 'magic number' for f.c.c. clusters. The next smallest model comprises 135 atoms in spherical shape of $D=$ $1.4 \mathrm{~nm}$. These two models turned into icosahedral structures during an initial MD run for relaxation. Another four larger models were assumed to be spherical, with 2-8 nm diameter. The largest model comprises 24499 atoms. Each model particle was set at the center of a cubic MD cell of $(12 \mathrm{~nm})^{3}$ with 3D periodic boundary conditions. The number of hydrogen atoms is 39559 . They were arranged initially in the face-centered cubic lattice within an octahedral space at the corner of the MD cell. ${ }^{12)}$ After starting MD calculation, hydrogen atoms evaporate rapidly from the octahedral crystal because of the $\mathrm{H}-\mathrm{H}$ repulsive forces and form a homogeneous gas phase outside the nanoparticles. The volume of the gaseous phase surrounding the nanoparticle varies about $15 \%$ according to the particle size. However such a small difference is considered negligible for simulation results.

The potential energy $E$ of the system is assumed to be in the form of 


$$
E=\frac{1}{2} \sum_{i j} V^{i-j}\left(r_{i j}\right)+\sum_{i} F\left(\rho_{i}\right),
$$

where $V^{i-j}$ denotes the pair potential between atoms $i$ and $j$ as a function of the distance $r_{i j}, F$ stands for the embedded function, and $\rho_{i}$ signifies the electronic density at atom $i$ from surrounding atoms. The metal-metal interaction, $V^{\mathrm{M}-\mathrm{M}}$, was fixed to that for f.c.c. Ni proposed by Ackland et al. ${ }^{16)}$ The atomic weight of metallic atoms was adjusted to Ni. The metal-hydrogen pair interaction, $V^{\mathrm{M}-\mathrm{H}}$, is assumed to be variable by introducing two parameters as $\varepsilon V^{\mathrm{M}-\mathrm{H}}(r+\Delta r)$ for parameter physics, ${ }^{12)}$ where $\varepsilon$ and $\Delta r$ respectively signify the energy and bond length parameters. As the reference function for $V^{\mathrm{M}-\mathrm{H}}$, the potential function proposed by Ruda et $a l .{ }^{17)}$ for the $\mathrm{Ni}-\mathrm{H}$ pair was adopted. The $\mathrm{H}-\mathrm{H}$ pair interaction is fixed to that with monotonic repulsion. ${ }^{17)}$ For simplicity, the embedded function only for $\mathrm{M}-\mathrm{M}$ pair was taken into account.

For parameter physics, $\varepsilon=1,2,4$, and 6 were assumed and $\Delta r$ was fixed to $-0.1 \mathrm{~nm}$. According to preliminary analyses, absorption energy of $\mathrm{H}$ atoms for these parameter values was estimated to be in the range of -21 to -176 $\mathrm{kJ} / \mathrm{mol} \mathrm{H}$, which corresponds to elements such as $\mathrm{Pd}, \mathrm{Th}, \mathrm{Ca}$, $\mathrm{Sr}$, and La. ${ }^{18)}$ The use of repulsive potential function for $\mathrm{H}-\mathrm{H}$ pair results hydrogen gas in the monoatomic phase. Therefore, we should limit our discussion to hydrogen dynamics inside the particle. The MD simulation was conducted using a constant-NVT condition at $300 \mathrm{~K}$ with velocity scaling for $100 \mathrm{ps}$, which is sufficient for equilibration of $\mathrm{H}$ atom in the case of $10 \mathrm{~nm}$ particles. ${ }^{13,14)}$ Calculation was conducted using a parallelized MD code ${ }^{19)}$ on a cluster computer composed of 192 cores.

\section{Results}

Figure 1 portrays lattice structures of hydrogenated nanoparticles with weak $(\varepsilon=1)$ and strong $(\varepsilon=6) \mathrm{M}-\mathrm{H}$ interactions. Five-fold symmetries were recognized in 1 and $1.4 \mathrm{~nm}$ particles. For weak $\mathrm{M}-\mathrm{H}$ interaction, $1 \mathrm{~nm}$ particles have a perfect icosahedral structure and $1.4 \mathrm{~nm}$ particles have a highly symmetric icosahedral arrangement lacking several surface atoms. The initial f.c.c. arrangement was maintained after hydrogenation in larger particles. These variations are commonly observed in hydrogen-free cases. For strong $\mathrm{M}-\mathrm{H}$ interaction, the resultant structures are slightly different. Icosahedral configurations in 1 and $1.4 \mathrm{~nm}$ models were disordered at the surface. Structures of $2 \mathrm{~nm}$ particle were strongly disordered, but its five-fold symmetries were apparently partly maintained. Such a shift of the critical size for icosahedral to cubic transition by hydrogenation was reported by Pundt et al. ${ }^{7)}$ The f.c.c. lattice was recognized in $4 \mathrm{~nm}$ and $6 \mathrm{~nm}$ models, but it was accompanied by lattice deformation or a grain boundary. Formation of the grain boundary by hydrogenation was also simulated in model b.c.c. nanoparticles. ${ }^{13,14)}$

Amounts of absorbed hydrogen atoms in nanoparticles are depicted in Fig. 2 for $\varepsilon=1,4$, and 6. The values of $\mathrm{H} / \mathrm{M}$ ratio vary from 0.6 to 1.9 as a function of cluster size and $\mathrm{M}-\mathrm{H}$ interaction. Generally, ratios increase concomitantly with increasing $\mathrm{M}-\mathrm{H}$ attractive interaction and particle size.

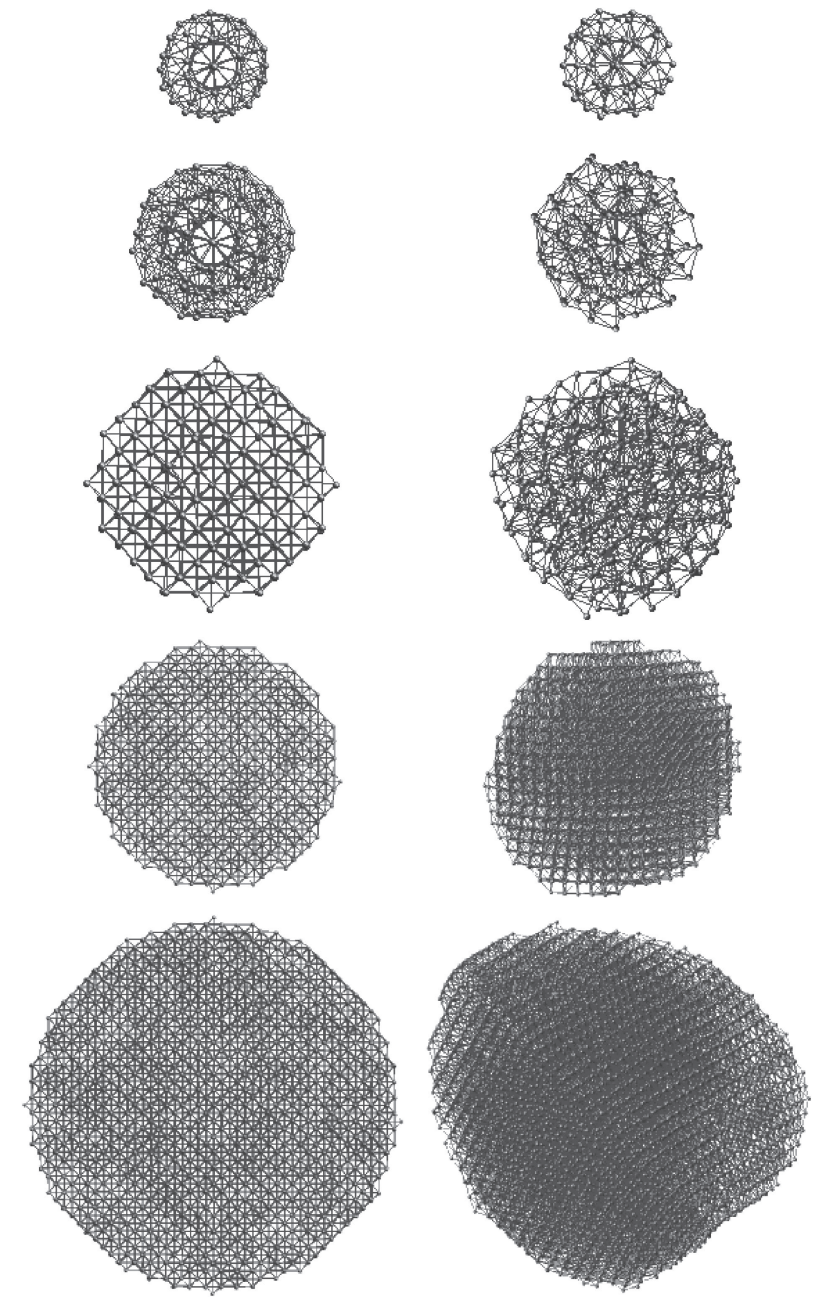

Fig. 1 Lattice structures after hydrogenation in model nanoparticles of $D / \mathrm{nm}=1$ (top), 1.4, 2, 4, and 6 (bottom) with the condition of $\varepsilon=1$ (left) and $\varepsilon=6$ (right). Figures of $D / \mathrm{nm}=4$ and 6 are scaled to half size. Hydrogen atoms are not shown here.

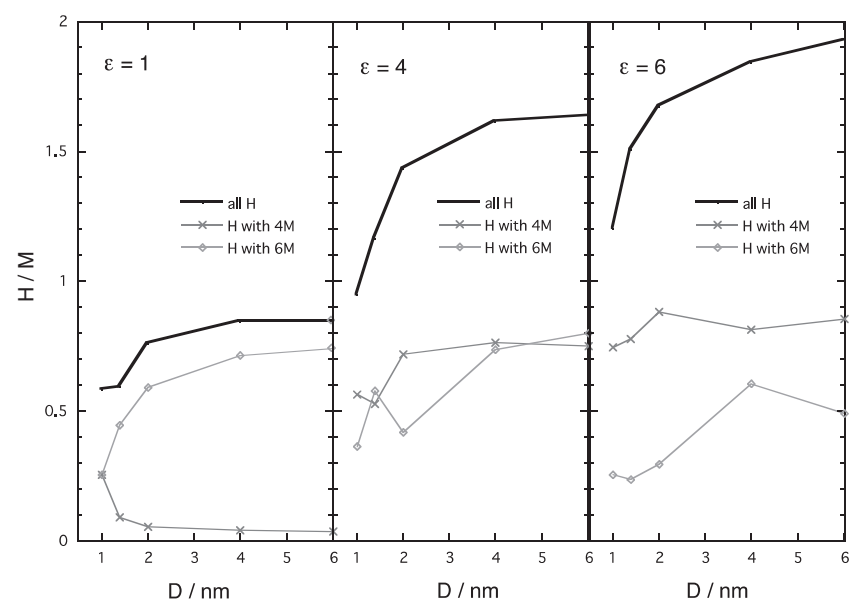

Fig. 2 The $\mathrm{H} / \mathrm{M}$ ratios of absorbed $\mathrm{H}$ atoms in nanoparticles with $\varepsilon=1,4$, and 6. Blue and red lines show the ratios of $\mathrm{H}$ atoms of which the respective coordination numbers of metallic atoms are 4 and 6.

Such positive dependency on particle size has been observed experimentally. ${ }^{10,11)}$ Numbers of hydrogen atoms coordinated by 4- 6 metallic atoms are also shown in the figure. In cases of $\varepsilon \leq 2$, about $80 \%$ of the absorbed hydrogen atoms 


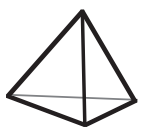

$\mathrm{T}$

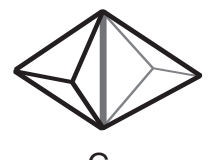

$\mathrm{C}_{2}$

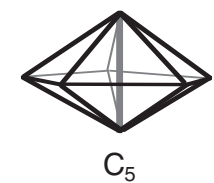

$\mathrm{C}_{5}$
Fig. 3 Three local structure units composed by metallic bonds analyzed in this study.
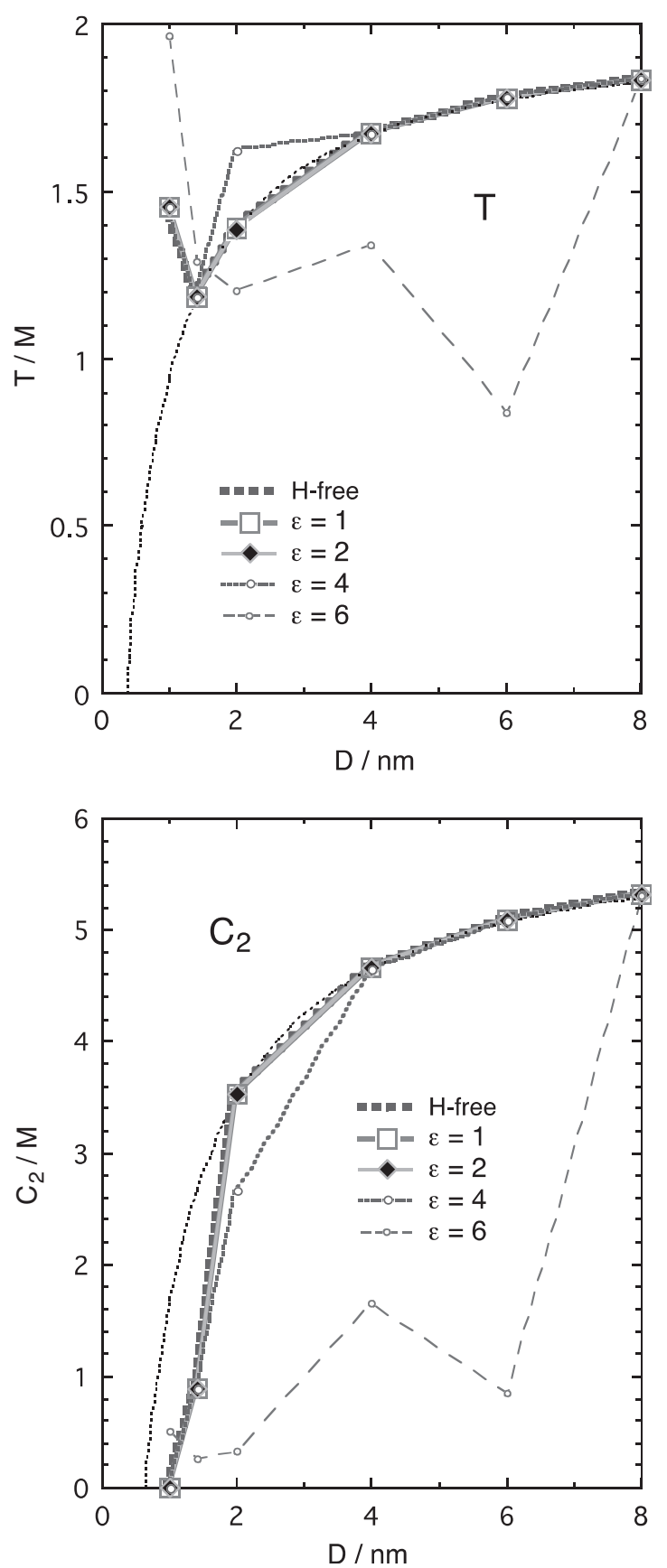

Fig. 4 Scaled number densities of the structure units $\mathrm{T}$ (top) and $\mathrm{C}_{2}$ (bottom) in hydrogen-free and hydrogenated nanoparticles as functions of particle diameter $D$. Dotted lines are the fitting curve with $r_{0}=0.18 \mathrm{~nm}$, $d_{\mathrm{s}}=0.18 \mathrm{~nm}(\mathrm{~T})$, and $r_{0}=0.31 \mathrm{~nm}, d_{\mathrm{s}}=0.18 \mathrm{~nm}\left(\mathrm{C}_{2}\right)$.

have six coordinating metallic atoms, which means that they locate mainly at $\mathrm{O}$-sites. The apparent small ratio of $\mathrm{H}$ atoms at $\mathrm{O}$-site in $1 \mathrm{~nm}$ particle is attributable to the limited number of O-sites. Quantities of $\mathrm{O}$ and $\mathrm{T}$ sites in 55 atoms icosahedral

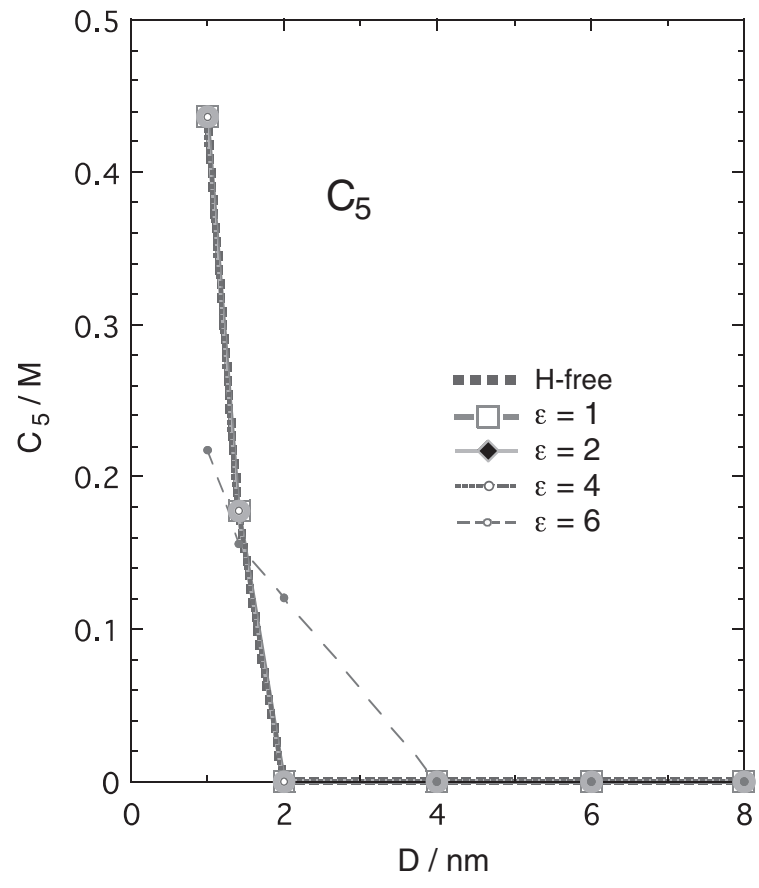

Fig. 5 Scaled number densities of the structure unit $\mathrm{C}_{5}$ in hydrogen-free and hydrogenated nanoparticles as functions of particle diameter $D$.

cluster are 20 and 80, respectively, which ratios are half of that in the f.c.c. bulk: $1: 2$. The actual number of $\mathrm{H}$ atoms at $\mathrm{O}$-site in this case is 14; consequently, the occupation ratio in $\mathrm{O}$-sites is $70 \%$. The number of hydrogen atoms at the T-site, however, increases with the $\varepsilon$ value. About half of the absorbed hydrogen atoms are located at T-site in the case of $\varepsilon=6$. Reduction of hydrogen atoms at O-site for high $\varepsilon$ values might be caused partly by lattice deformation observed in Fig. 1, which generates asymmetric interstitial sites having coordination numbers of 5 or 7 .

\section{Discussion}

As presented above, hydrogenation of small f.c.c. particles induces complex structural variation depending on both particle size and $\mathrm{M}-\mathrm{H}$ interaction. To understand the hydrogenation mechanism, local structures of metallic lattice in nanoparticles were analyzed. Figure 3 presents three local units named $\mathrm{T}, \mathrm{C}_{2}$, and $\mathrm{C}_{5}$ used in this analysis. The $\mathrm{T}$ unit is a tetrahedron that is not necessarily a regular tetrahedron as in the f.c.c. lattice. The $\mathrm{C}_{2}$ unit comprises two tetrahedra connected by one common edge that appears everywhere in the f.c.c. lattice. The $\mathrm{C}_{5}$ unit is a combination of five tetrahedral with one common edge which does not exist in f.c.c. lattice, but which appears in icosahedral clusters and possibly in amorphous metals.

Data related to $\mathrm{T}$ and $\mathrm{C}_{2}$ units in hydrogen-free and hydrogenated nanoparticles are given in Fig. 4. The number of $\mathrm{T}$ and $\mathrm{C}_{2}$ in hydrogen-free or low $\varepsilon$ cases is known to converge to a smooth, monotonically increasing curve. The values scaled by the number of metallic atoms $M$ approach those of bulk, 2 and 6 for $\mathrm{T}$ and $\mathrm{C}_{2}$, respectively. Herein, we consider a trial form for these curves as $\left(r-r_{0}\right) /\left(r-d_{\mathrm{s}}\right)$ where $r$ is the radius of nanoparticle and $r_{0}$ and $d_{\mathrm{s}}$ are fitting parameters respectively representing the minimal radius for 
which $\mathrm{T}$ or $\mathrm{C}_{2}$ vanishes, and the surface layer thickness. As dotted lines in the figure show, the trial form expresses the variations of these units, except for smaller particles in which icosahedral transition occurs. Deviations from the fitting curves were observed in cases of $2 \mathrm{~nm}$ particle for $\varepsilon=4$ and all particle sizes for $\varepsilon=6$. Figure 1 shows that these deviations result from the lattice deformation. Therefore, it can be summarized that the lattice structure is affected by surface effects and the icosahedral transition in hydrogenfree nanoparticles, and also by lattice deformation attributable to $\mathrm{M}-\mathrm{H}$ interaction in hydrogenated nanoparticles.

To separate the effects of icosahedral transformation and lattice deformation, structure unit $\mathrm{C}_{5}$ was analyzed as presented in Fig. 5. If the 55 atoms cluster has an ideal icosahedral structure, there exist $24 \mathrm{C}_{5}$ units hence the $\mathrm{C}_{5} / \mathrm{M}$ ratio should be 0.44 . The current result shows good agreement with this assumption. The $\mathrm{C}_{5}$ units were observed in very narrow ranges in $D<2 \mathrm{~nm}$. The existence of partial icosahedral symmetries remaining in $2 \mathrm{~nm}$ particle for $\varepsilon=6$ observed in Fig. 1 is confirmed here. Consequently, we conclude that icosahedral transformation attributable to hydrogenation occurs in some cases, and that effects of hydrogenation in particles larger than $2 \mathrm{~nm}$ take places mainly as lattice deformation and grain boundary generation, as presented in Fig. 1.

\section{Summary}

Hydrogen absorption and related structure variation in f.c.c. nanoparticles were investigated using classical MD simulation. Transformation from f.c.c. to icosahedral arrangements was observed in both hydrogen-free and hydrogenated particles smaller than $2 \mathrm{~nm}$. The fundamental structure in larger particles was found to be f.c.c. with some modification by lattice deformation and the grain boundary. The amount of absorbed hydrogen atoms increased concomitantly with increasing particle size and $\mathrm{M}-\mathrm{H}$ interaction. About $80 \%$ of $\mathrm{H}$ atoms located at $\mathrm{O}$-sites in cases of weak $\mathrm{M}-\mathrm{H}$ interaction, and the ratio of $\mathrm{H}$ at $\mathrm{T}$-site increased concomitantly with increasing $\mathrm{M}-\mathrm{H}$ interaction. Analysis based on the local structure units showed that structural variation in nanoparticles can be understood by surface effects, icosahedral transformation, and lattice deformation attributable to $\mathrm{M}-\mathrm{H}$ interaction. The surface effect was expressed as a simple trial form. The current MD simulation, which presented a specific image of hydrogenation of nanoparticles, is expected to provide valuable information for elucidating the hydrogen storage mechanism by nanoparticles.

\section{Acknowledgement}

This work has been supported by New Energy and Industrial Technology Development Organization (NEDO) under "Advanced Fundamental Research Project on Hydrogen Storage Materials".

\section{REFERENCES}

1) T. Mutschele and R. Kirchheim: Scr. Metall. 21 (1987) 1101-1104.

2) U. Stuhr, H. Wipf, T. J. Udovic, J. Weissmuller and H. Gleiter: J. Phys. Cond. Matter 7 (1995) 219-230.

3) L. Zaluski, A. Zaluska and J. O. Ström-Olsen: J. Alloy. Compd. 253 (1997) 70-79.

4) H. Natter, B. Wettmann, B. Heisel and R. Hempelmann: J. Alloy. Compd. 253 (1997) 84-86.

5) A. Pundt, C. Sachs, M. Winter, M. T. Reetz, D. Fritsch and R. Kirchheim: J. Alloy. Compd. 293 (1999) 480-483.

6) M. Suleiman, N. M. Jisrawi, O. Dankert, M. T. Reetz, C. Bähtz, R. Kirchheim and A. Pundt: J. Alloy. Compd. 356 (2003) 644-648.

7) A. Pundt, M. Dornheim, M. Guerdane, H. Teichler, H. Ehrenberg, M. T. Reetz and N. M. Jisrawi: Euro. Phys. J. D 19 (2002) 333-337.

8) T. D. Klots, B. J. Winter, E. K. Parks and S. J. Riley: Chem. Phys. 95 (1991) 8919-8930.

9) A. Pundt and R. Kirchheim: Ann. Rev. Mater. Res. 36 (2006) 555-608.

10) M. Yamauchi, R. Ikeda, H. Kitagawa and M. Takata: J. Phys. Chem. C 112 (2008) 3294-3299.

11) M. Yamauchi, H. Kobayashi and H. Kitagawa: Chem. Phys. Chem. 10 (2009) 2566-2576.

12) H. Ogawa, A. Tezuka, H. Wang, T. Ikeshoji and M. Katagiri: Mater. Trans. 49 (2008) 1983-1986.

13) H. Ogawa, A. Tezuka, H. Wang, T. Ikeshoji and M. Katagiri: Int. J. Nanosci. 8 (2009) 39-42.

14) H. Ogawa, M. Kayanuma and M. Katagiri: MRS Symp. Proc. 1216E (2010) W03-02.

15) H. Ogawa: J. Alloy. Compd. in press.

16) G. J. Ackland, G. Tichy, V. Vitek and M. W. Finnis: Phil. Mag. A 56 (1987) 735-756.

17) M. Ruda, D. Farkas and J. Abriata: Phys. Rev. B 54 (1996) 9765-9774.

18) R. Griessen and A. Driessen: Phys. Rev. B 30 (1984) 4372-4381.

19) H. Ogawa: Mater. Trans. 48 (2007) 2067-2071. 